\title{
Design and Fabrication of Pneumatic Sheet Metal Cutting and Punching Machine
}

\author{
Aryan Pratihar \\ Department of Mechanical Engineering \\ Jss Academy of Technical Education \\ Noida, India \\ Ayog Kumar Rastogi \\ Department of Mechanical Engineering \\ Jss Academy of Technical Education \\ Noida, India
}

\author{
Adarsh Raunak \\ Department of Mechanical Engineering \\ Jss Academy of Technical Education \\ Noida, India \\ Aakash Sharma \\ Department of Mechanical Engineering \\ Jss Academy of Technical Education \\ Noida, India
}

\author{
Vikas Kumar Singla \\ Department of Mechanical Engineering \\ Jss Academy of Technical Education \\ Noida, India
}

\begin{abstract}
Metal in the form of sheets is paramount in the manufacturing industry. Its applications are countless. But metal in the form of sheets cannot be directly used, operations like cutting, punching, blanking, bending, trimming, etc. are needed to be carried out on the metal sheets in order to fully utilize them. For these operations, most large scale manufacturing industries use hydraulically operated machines. But since hydraulic machines are not cost-effective, most small and medium scale industries use hand-operated machines for carrying out sheet metal operation. The problem with hand-operated machines is that they are slow and cannot be automated. This is where the concept of pneumatics will prove itself advantageous. We are developing a pneumatically operated cutting and punching machine which will use the help of compressed air to drive a shearing blade and punch to carry out the operations on a metallic sheet. The machine is designed to cut $1 \mathrm{~mm}$ thick aluminium sheets and $0.5 \mathrm{~mm}$ thick copper sheets very easily. The main components of developed pneumatic system are compressor, pipelines, double-acting cylinder, an actuator, and flow control valves. This machine will thus, prove itself cost-effective and also save a lot of time in manufacturing process as two operations can be performed on the same platform.
\end{abstract}

Keywords: Pneumatic, sheet, cutting, punching, clearance, industry, aluminium, compressor

\section{INTRODUCTION}

The production of iron and steel is of great importance for any country's economy. This is because the iron and steel industry directly influences the development of a sustainable society and also it the basis for all other industries. Thus the trend in production and consumption in steel is also viewed as an indicator of the condition of the country's economy. This the reason why steel is called the "backbone" of a country's economy. Hence by increasing the production of steel, we can help in the growth of our country's economy. But this where the problem arises. A sheet metal cutting or a punching machine is very important to the sheet metal industry and since large scale industries are well established they can afford to equip themselves with hydraulically operated cutting and punching machines that generate a large amount of force and also are easily automated. Thus the production output of large scale industries is enormous. Same is not the case with medium to small scale industries. Since hydraulic machines are too costly most of these industries restrict themselves to only using hand-operated cutting or punching machines. The production output of handoperated machines is low. Because of this problem, we are developing a pneumatically operated cutting and punching machine. Pneumatics is the branch of engineering which uses pre-compressed air or inert gas as a means to drive machinery. Certain properties of air make it extremely suitable for its usage in modern machinery.

The advantage of using a pneumatic system is that it can generate a considerable amount of force while being costeffective.

\section{LITERATURE REVIEW}

Madhukumar V. et. al. [1] developed a pneumatic machine that would perform cutting as well as bending operation to reduce the cost of operations performed on sheet metal. Further modifications in their design can also increase the cutting force.

T. Z. Quazi et. al. [2] studied the influence of punch-die clearance in blanking process. Their investigation showed that by decreasing clearance the required blanking force increased. They found that $10 \%$ is the optimum clearance is required for minimizing blanking force.

Viraj N. Suryawanshi et. al. [3] fabricated a pneumatic punching machine to reduce punching cost on metallic sheet.

K. K. Alaneme et. al. [4] investigated the reasons behind the failure of mould dies in a punching machine. They found out that die failure happened because of improper heat treatment thus reducing the toughness and fatigue resistance of die material.

Neeraj Pandita et. al. [5] developed a pneumatic sheet metal cutting machine which is better in comparison to 
manual driven sheet cutters. The efficiency of the cutter can be increased by further enhancement in cutting blade. Sudeep Kelaginamane et. al. [6] designed a PLC controlled pneumatic punching machine. The machine reduced the production time and increased productivity from 60 units per hour to 420 units per hour.

A. K. Gupta et. al. [7] studied the influence of parameters like blanking force, clearance, blanking layout on sheet deformation. They found that the tolerance in the dimension of the punch hole can be minimized by increasing the compressor pressure. They found that variation in the dimension was more in case of a galvanized iron sheet as compared to the aluminium sheet.

T. Jon Babu et. al. [8] found out that by changing the pressure of compressed air variable cutting forces could be obtained and by adding more accessories this equipment can cut a higher range of sheet thickness

Arun S. et. al. [9] developed a method for controlling the operations of punching machine using Programmable Logic Controllers. Reduced manufacturing lead time and increase worker safety using this system.

A. S. Aditya Polapragada et. al. [10] developed a pneumatic and punching machine. The project helped reduce manufacturing cost for small scale industries.

R. M. Lathe et. al. [11] converted a manually controlled press into an automatic machine using which they saved maximum operating time. Using this maximum output increase and human intervention decreased.

Pradeshi Ram et. al. [12] converted a manually operated conventional sheet bending machine to an automatic machine and eliminated the problem of signal overlapping by using stepper module.

Indrajeet Chaudhary et. al. [13] employed a stepper module, a component of advanced pneumatics for automation of conventional sheet metal bending machine operations thus converting a manually operated bending machine to an automatic machine.

Khagendra Barman et. al. [14] developed a pneumatic sheet metal cutting machine which runs by means of precompressed air. It is an efficient way of increasing production for small scale industries.

Suleyman Yaldiz et. al. [15] developed a pneumatic accelerator for high-speed punching which can be easily employed in conventional presses thus eliminating the use of high energy rate forming (HERF) machines to store energy. The accelerator when employed on mechanical presses converts low speed operation of hammer to high speed operation.

F. W. Travis et. al. [16] experimented on the high speed perforation of mild steel plates for impact velocities upto $300 \mathrm{~m} / \mathrm{s}$ analysed the bulge height at perforation. They concluded with the result that bulge height increased with the increase of plate thickness but due to the development of thermo-plastic instability it dropped subsequently.

Karan Dutt et. al. [17] studied various types of pneumatic machines and components along with their advantages and disadvantages. He concluded that pneumatic machines can provide power at a cheaper, safer and more reliable way than electric motors and actuators.
A. K. Murthy et. al. [18] designed and fabricated mechanically operated paper shearing machine with the capacity to cut $25 \mathrm{~mm}$ thick and $300 \mathrm{~mm}$ wide paper. The machine could produce a variable force using screw press which made it useful to perform other operations like bending, punching and embossing.

Martin Feistle et. al. [19] developed methods to measure the formation of edge cracks on shearing blades. It has been found that the forming strength of high strength steels is curtailed due to edge fractures and can be improved by varying process parameters like die clearance, geometry and cutting line.

Utkarsh Sharma et. al. [20] designed and fabricated an automatic pneumatic hole punching machine powered by solar energy. The machine has been designed on SolidWorks software. Since the machine uses solar energy as a source of energy it will eliminate/reduce the usage of electicity in running the machines in small scale industries.

\section{CONCLUSION}

It is observed that pneumatic machines are cheaper than hydraulic machines. Cost of maintenance is also low as compared to hydraulic machines since compressed air is the working fluid. The force required for punching and shearing machines and the tool wear can be controlled by changing the process variables like clearance and angle. 'Pneumatic Sheet metal Cutting and Punching Machine' is advantageous as it can be afforded by small scale industries. Since two operations, that is cutting and punching, can be performed on the same platform, it significantly reduces the production time.

\section{REFERENCE}

[1] Madhukumar V, Arun Kumar N, Harsha B S, Navin Kumar K N, Nagaraja T.K.2 International Journal of Engineering Research and Advanced Technology (IJERAT) ISSN: 2454-6135 [Special Volume. 02 Issue, 01, May-2016].

[2] T. Z. Quazi, R.S.Shaikh, "An Overview of Clearance Optimization in Sheet Metal Blanking Process" International Journal of Modern Engineering Research (IJMER) Vol.2, Issue.6, Nov-Dec. 2012 pp-4547-4558 ISSN: 2249-6645.

[3] Viraj N. Suryawanshi, Nilesh V.Wakade, Prof. Prashant A. Narwade "Design and Development of Pneumatic Punching Machine" International Research Journal of Engineering and Technology (IRJET) e-ISSN: 2395-0056 Volume: 06 Issue: 05 May 2019 p-ISSN: 2395-0072

[4] K.K. Alaneme, B.O. Adewuyi, F.A. Ofoegbu "Failure analysis of mould dies of an industrial punching machine"

[5] Neeraj Pandita, Naren Kesar, Akshit Jasrotia, Surya Dev Singh, Lakshay Jolly, Anant Khajuria , Vishwarth Singh , "Pneumatic Sheet Cutting Machine- A Review" International Journal of Scientific and Technical Advancements ISSN: 2454-1532

[6] Sudeep Kelaginamane, Sridhar D. R. "PLC Based Pneumatic Punching Machine" Journal of Mechanical Engineering and Automation.

[7] A.K. Gupta, P. Bharadwaj, S. Sahgal, P. M. Pradhan, "Experimental Investigation and Fabrication of Pneumatic Punch", International Journal of Innovative Research in Science, Engineering and Technology, Volume 2, Issue 6, June 2013.

[8] M.Khaja Gulam Hussain, T. John babu, "Fabrication of Pneumatic Shearing Machine”, 2016 IJRTI | Volume 1, Issue 2 | ISSN: 2456-3315

[9] Arun S, Sree Rajendra, Vijayavithal Bongale, "Automatic Punching Machine: A Low Cost Approach", International Journal of Advanced Mechanical Engineering ISSN 2250-3234 Volume 4, Number 5 (2014), pp. 509-517 
[10] Shubhkumar Bhandari, Rajkumar B. Chadge, " Methodology of Special Purpose Sheet Metal Cutting Machine", IJPRET, 2014; Volume 2 (9): 1-8 ISSN: 2319-507X

[11] Aditya Polapragada, Sri Varsha, "Pneumatic Auto Feed Punching and Riveting Machine", International Journal of Engineering Research \& Technology (IJERT), Vol. 1 Issue 7, September 2012, ISSN: 2278-0181.

[12] Vijaylaxmi G. Biradar, Siddharam Patil , R M Lathe, "Automation of Sheet Bending Machine Using Electro Pneumatic Devices" International Journal of Scientific \& Engineering Research Volume 3, Issue 9, September-2012 ISSN 2229-5518.

[13] Indrajeet Chaudhary, Pradeshi Ram, Dinesh Kumar, "Using Advanced Pneumatics Automation of Conventional Sheet Bending Machine" IJISET, Vol. 1 Issue 9, November 2014

[14] Khagendra Barman, Md. Nesar Ali, Md Rayhan Hasnat, Dr S. M. Humayun Kabir, "Fabrication of a Pneumatic Sheet Metal Cutting Machine".

[15] Suleyman Yaldı, Hacı Saglam, Faruk Unsac,ar, Hakan Isßık, "Design and applications of a pneumatic accelerator for highspeed punching"
[16] A. I. O. Zaid, F. W. Travis "An Examination Of The Effect Of Target Thickness In The Perforation Of Mild Steel Plate By A Flat-Ended Projectile" Int. J. meek. Sci. Pergamon Press. 1974. Vol. 16, pp. 373-383. Printed in Great Britain.

[17] Karan Dutt, "Analytical Description of Pneumatic System", International Journal of Scientific \& Engineering Research, Volume 4, Issue 9, September-2013 1443 ISSN 2229-5518

[18] A. K. Murthy, Amith V., B.M. Satish, Nikhil S., Nagaraj, Pradosh Kumar Sahu, Rohit Raj, " Mechanically Operated Paper Shearing Machine", International Journal of Innovative Research in Science, Engineering and Technology (An ISO 3297: 2007 Certified Organization) Vol. 5, Issue 5, May 2016.

[19] Martin Feistel, Roland Golle, Wolfram Wolk, "Determining the influence of shear cutting parameters on the edge cracking susceptibility of high-strength-steels using the edge-fracturetensile-test" , 48th CIRP Conference on MANUFACTURING SYSTEMS - CIRP CMS 2015

[20] Utkarsh Sharma, "Design of Automatic Pneumatic Hole Punching Machine", International Research Journal of Engineering and Technology (IRJET), Volume: 02 Issue: 09 | Dec-2015. 